\title{
Effect of exogenous gonadotropin on the transcriptome of human granulosa cells and follicular fluid hormone profiles
}

Cui-Ling Lư 1,2,3,4, Zhi-Qiang Yan ${ }^{1,2,3,5}$, Xue-Ling Song ${ }^{1,2,3,4}$, Yang-Ying $X u^{1,2,3,4}$, Xiao-Ying Zheng ${ }^{1,2,3,6}$, Rong Li $i^{1,2,3,6}$, Ping Liu ${ }^{1,2,3}$, Huai-Liang Feng ${ }^{7^{*}}$ and Jie Qiao ${ }^{1,2,5,6^{*}}$

\begin{abstract}
Background: Superovulation treatment had some adverse effects on maturity and development of oocytes. Can superovulation dose of gonadotropins (Gns) affect the transcriptome of granulosa cells and follicular fluid (FF) hormone levels?

Methods: One leading pre-ovulatory follicle per subject was used from three natural-cycle and four Gn-stimulated patients. Granulosa cells and FF samples were collected from the same leading follicle of each patient. RNA was extracted from granulosa cells and subjected to deep sequencing and analysis. Follicle-stimulating hormone (FSH), estradiol (E2), androstenedione (AND), testosterone (T), luteinizing hormone (LH), and progesterone (P4) levels in FF were measured by immunoassays. Student's $t$ test was used for statistical analysis.

Results: A total of 715 genes were up-regulated, and 287 genes were down-regulated, in the Gn-stimulated group relative to the control group. Gene Ontology analysis revealed that the down-regulated genes were enriched in cell cycle and meiosis pathways, primarily those associated with follicle or oocyte maturation and quality. On the other hand, the up-regulated genes were enriched in functions related to immunity and cytokine-cytokine receptor interactions. Compared to the follicles of natural cycle, the E2 and LH concentrations were significantly reduced $(P<0.001)$, the $\mathrm{P} 4$ concentration was significantly increased $(P=0.003)$, and the concentrations of FSH, T and AND had no difference in the follicles of Gn-stimulated cycle.

Conclusions: Cell cycle- and meiosis-associated genes were down-regulated by Gns stimulation, whereas immuneand cytokine-associated genes were up-regulated. Hormone levels were also affected by Gns stimulation. Compared with natural-cycle follicles,putative markers associated with oocyte quality and follicle maturation were significantly different from those in Gn-stimulated follicles. Hormone levels in follicles were compatible with the steroidogenic patterns of granulosa cell, which reflects the follicle maturation and oocyte quality.
\end{abstract}

Keywords: Granulosa cells, Transcriptome, Follicular fluid, Gonadotropins, Natural cycle

\section{Background}

Controlled ovarian stimulation (COS) using exogenous gonadotropins (Gns) is a conventional step of the in vitro fertilization (IVF) treatment. With regard for this technique, oocyte retrieval is performed with an aim to

\footnotetext{
* Correspondence: doctorf88@gmail.com; jie.qiao@263.net

${ }^{7}$ Department of Obstetrics and Gynecology, New York Hospital

Queens-affiliated Weill Medical College, Cornell University, New York 10041NY212, USA

${ }^{1}$ Center for Reproductive Medicine, Department of Obstetrics and Gynecology, Peking University Third Hospital, Beijing 100191, China Full list of author information is available at the end of the article
}

harvesting a large number of oocytes to achieve a successful pregnancy (i.e., the more oocytes obtained per cycle, the more embryos can be selected for transfer) [1]. In recent years, the concept of acquiring as many oocytes as possible has been shifted to place emphasis on obtaining a cohort of high-quality embryos [2]. Although various COS protocols have been proposed to achieve a large number of oocytes and subsequently developed embryos, there is no evidence to support an appropriate and optimal protocol that obtains the best quality of products.

(C) The Author(s). 2019 Open Access This article is distributed under the terms of the Creative Commons Attribution 4.0 International License (http://creativecommons.org/licenses/by/4.0/), which permits unrestricted use, distribution, and 
Additionally, COS may result in the recruitment of oocytes that are not reaching their optimal maturation or full competency, and thus are not the best quality of oocytes and embryos compared to those obtained via the natural cycle [3-5]. Studies performed using clinical samples have demonstrated that compared with conventional IVF cycle, treatment using in natural cycle IVF without embryo selection achieved a higher implantation rate $[6,7]$. Furthermore, ovarian stimulation with a higher dose of Gns can lead to an iatrogenic complication called ovarian hyperstimulation syndrome (OHSS), which can be life-threatening. Moreover, offspring of women who experience OHSS has been reported to exhibit a higher chance of diminished intellectual ability and cardiovascular dysfunction $[8,9]$.

Collectively, IVF performed using natural cycle protocol may provide a superior environment for oocyte maturation. Therefore, the application of COS protocol for IVF may have a detrimental effect on the follicular milieuand hence an impact on the maturation process and developmental competence of oocytes. In this study, we aimed to investigate the endocrine milieu in the naturecycle follicle and the COS-follicle by comparing the granulosa cells and hormonal composition in the follicle fluid samples of two conditions during the time of follicular aspiration.

\section{Methods}

\section{Study design, participants and collection of granulosa cells}

The study was conducted at the Reproductive Medical Center of the Third Hospital Peking University (Beijing, China). All the recruited patients were under 36 years old and had a normal BMI range from 18.8 to $26.6 \mathrm{~kg} / \mathrm{m}^{2}$. These patients received either the Gn stimulation protocol (the same COS protocol) or natural cycle for their IVF treatment, as explained below. The exclusion criteria were patients with the ovulatory disorder or follicular dysplasia. Four women who received the COS protocol for IVF treatment had a regular menstrual cycle because of male factor or tubal factor. For these patients, a combination of recombinant follicle stimulating hormone (FSH) (Gonal-F, 150 IU/day, Merck Serono SA Aubonne Branch) and human menopausal gonadotrophin (Menotropins for injection FSH 75 IU: LH 75 IU, Livzon Pharmaceutical Group Inc.) in a fixed-dose was started on Day 2 of the menstrual cycle with the option to adjust dose according to response after 4 days of stimulation (Day 6 of menstrual cycle). GnRH antagonist (Cetrorelix $250 \mu \mathrm{g} /$ day, Merck Serono, Darmstadt, Germany) was started when a leading follicle of $12 \mathrm{~mm}$ was achieved. Moreover, when one or two leading follicles reached an average diameter of $18 \mathrm{~mm}$, the recombinant human chorionic gonadotrophin (hCG) (Ovitrelle, $250 \mu \mathrm{g}$;
Merck Serono S.p.A, Rome, Italy) was administered followed by follicular aspiration 34-36 h later. These patients were assigned as the $\mathrm{Gn}$-stimulated group $(n=4)$. In the unstimulated-cycle group (assigned as the control group, $n=3$ ), three patients were arranged for IVF treatment with their natural cycles without $\mathrm{Gn}$ intervention and with no hCG triggering. These infertile women were due to tubal factors, including hydrosalpinx and proximal tubal obstruction. In both groups, the follicular fluid samplesobtained from the largest pre-ovulatory follicle of each patient were collected for analysis. The technique of follicular aspiration was performed with a new 17-gauge singlelumen aspiration needle (K-OPS-7035-REH-ET; Cook, Queensland, Australia) and a suction pressure of 120 $\mathrm{mmHg}$, under the guidance with transvaginal ultrasonography. Immediately after the aspirates were collected, we centrifuged these aspirates at $2000 \mathrm{Xg}$ for $10 \mathrm{~min}$, and the supernatant was transferred to a 2-ml cryotube for cryopreservation at $-80^{\circ} \mathrm{C}$ until later analysis. The sediment samples were collected for granulosa cell isolation, which were further washed in phosphate-buffered saline (PBS) and centrifuged over Ficoll (GE Healthcare Corp., USA) to remove the red blood cells. The aggregates that contain granulosa cells were isolated from the follicular fluid by a pipette, flushed twice in phosphate-buffered saline (PBS), and transferred to a tube placed in ice water. Granulosa cells were washed again with PBS, and the cell deposits were flash frozen in liquid nitrogen within $30 \mathrm{~min}$ and stored at $-80^{\circ} \mathrm{C}$ until RNA extraction.

\section{Preparation of cDNA from a small number of cells and PCR pre-amplification}

The experimental protocol for cDNA preparation and PCR pre-amplification was as previously described [10]. Briefly, deposits of a small amount of granulosa cells were transferred into lysis buffer and reversely transcribed into the first-strand cDNA. The cDNA was then amplified by a PCR machine for 20 cycles.

\section{Tagmentation reaction and final PCR amplification}

PCR was purified and ultimately eluted. Five nanograms of cDNA were then used for the tagmentation reaction, purified, and then used for second PCR amplification. The PCR products were purified using an AMPure XP kit (Beckman Coulter, Brea, CA, USA), and samples were loaded onto an Agilent Bioanalyzer 2100 system (Agilent Technologies, Santa Clara, CA, USA). Quantification of the library was performed using the Qubit 3.0 Fluorometer (Thermo Fisher Scientific, Singapore). Libraries were diluted to a final concentration of $2 \mathrm{nM}$, and a total of 10 pmol of each library was sequenced using an Illumina HiSeq 2000. 


\section{Read alignments and estimation of gene expression}

Clean data (clean reads) were obtained by removing artificial adapters, poly-A, and low-quality bases from raw data. Clean data were aligned to human (hg19) genome using Tophat2 (v2.1.0) with default settings and filtered for uniquely mapped reads. Gene expression values were calculated as FPKM using Cufflinks (v2.2.1) [11].

\section{Data analysis}

Differential expression analysis of the two groups was performed using the DEGSeq R package (v1.18.0). DEGSeq analysis was used to provide statistical routines for determining differentially expressed genes (DEGs) using a model based on the negative binomial distribution. $P$-values were adjusted using the Benjamini-Hochberg method to control the false discovery rate. Genes with adjusted $P$-value $<0.05$ were defined as differentially expressed.

Gene Ontology (GO) enrichment analysis was performed using the GOseq $\mathrm{R}$ package with correction for gene length bias. GO terms with corrected $P$-values less than 0.05 were considered to be significantly enriched. Enrichment of DEGs in the Kyoto Encyclopedia of Genes and Genomes (KEGG) was analyzed using the KOBAS software [12].

\section{Hormone assay procedures}

All hormonal assays were performed at the endocrine laboratory of the Peking University Third Hospital Reproductive Centre using commercially available ELISA kits (IMMULITE 2000, SIEMENS, USA). The lower detection limits of the six assays used in this study were as follows: $0.1 \mathrm{mIU} / \mathrm{ml}$ for $\mathrm{FSH}, 0.1 \mathrm{mIU} / \mathrm{ml}$ for luteinizing hormone (LH), $73.4 \mathrm{pmol} / \mathrm{l}$ for estradiol (E2), $0.64 \mathrm{nmol} / \mathrm{l}$ for progesterone (P4), $0.69 \mathrm{nmol} / \mathrm{l}$ for testosterone $(\mathrm{T})$, and 1.05 $\mathrm{nmol} / \mathrm{l}$ for androstenedione (AND), respectively. The inter-assay coefficients of variation (CVs) for the six hormone levels were 5\% (FSH), 6\% (LH), 5\% (E2), 6\% (P), 6\% (T), and 5\% (AND), respectively.

\section{Statistical analysis}

Hormonal concentration valuesare expressed as means \pm SD (standard deviation). Differences in hormone concentration between the two groups were analyzed by student's t test.

\section{Results}

\section{Samples and sequencing}

The characteristics of the patients of the control group $(n=3)$ and $\mathrm{Gn}$-stimulated group $(n=4)$ were as the previous described in the Materials and Methods section. Transcriptomes of the individual luteinized granulosa cells from every single pre-ovulatory follicle obtained from these seven women were sequenced.
RNA transcriptomes were sequenced using the Illumina HiSeq platform. We obtained 69-90 million 150 bp reads for each sample, and $76 \mathrm{~Gb}$ of raw data in total were obtained for all of the samples. Clean data were $10 \mathrm{~Gb}$ per sample (Additional file 2: Table S1). All the downstream analyses were conducted based on clean, high-quality data.

\section{Transcriptional profiles between different samples}

We calculated the Pearson correlation coefficient of every two samples by using the gene expression in the samples. As shown in Additional file 1: Figure S1A, the expression patterns were generally homogeneous among the three controls $\left(R^{2}>0.9\right.$ for both comparisons). In contrast, the expression patterns were relatively heterogeneous in the Gn-stimulated group, with Gns1 and Gns3 $\left(R^{2}=0.917\right)$, and Gns2 and Gns4 $\left(R^{2}=0.841\right)$, are more similar to each other.

We then examined the total number of transcripts that were expressed in each group. The results obtained from the comparison analyses revealed that 11,923 genes were commonly expressed in both groups in which 2437 genes were expressed uniquely in the Gn-stimulated group and 920 genes were uniquely expressed in the control group (Additional file 1: Figure S1B).

\section{DEGs and function enrichment analysis}

We then analyzed the DEGs between the two groups. As shown in Fig. 1a, we came up with the unsupervised hierarchical clustering of DEGs. Compared with the control group, 715 genes were up-regulated, whereas 287 genes were down-regulated in the Gn-stimulated group $\left(P<0.05\right.$; FC of $\log _{2}$-transformed FPKM $\left.>1\right)$ (Fig. 1b). The detailed regarding genes that were up-regulated and down-regulated in each group are presented in Additional file 3: Table S2 and Additional file 4: Table S3.

To investigate the possible biological functions of DEGs between the two groups, we performed GO analysis of DEGs and identified enriched biological processes in each group (the detailed information is presented in Additional file 5: Table S4 and Additional file 6: Table S5). The top $15 \mathrm{GO}$ enrichment results are shown in Fig. 2a (the down-regulated DEGs) and Fig. 2b (the up-regulated DEGs). The DEGs down-regulated in the Gn-stimulated group were significantly enriched and related to the cell cycle and chromosome segregation. In contrast, the up-regulated DEGs were enriched in functions that are related to immune response or processes.

Next, we performed the KEGG analysis to investigate the differences between the two groups. Detailed pathway enrichment analyses of down-regulated and upregulated DEG data are presented in Additional file 7: Table S6 and Additional file 8: Table S7, respectively. The representative top 10 pathways $(P<0.05)$ in DEGs 
A

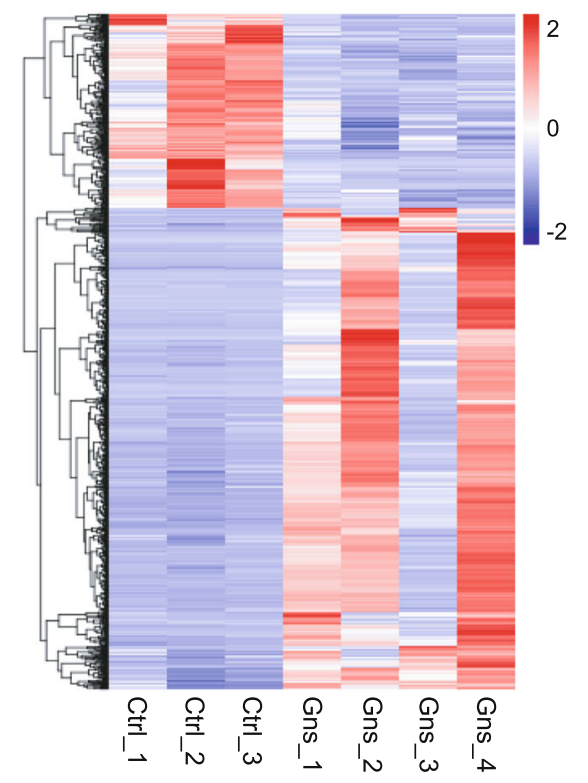

B

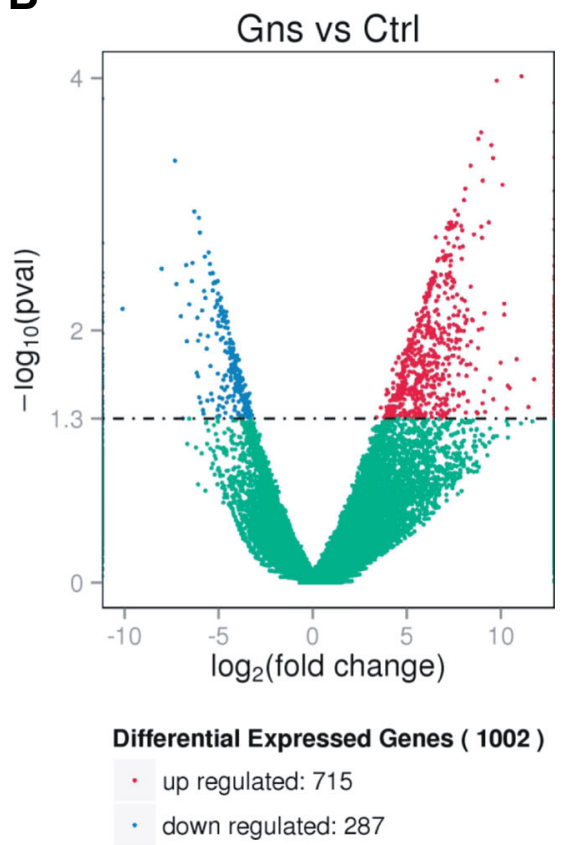

Fig. 1 Cluster and filter analysis of DEGs. a Heatmap of the differentially expressed genes (DEGs) between Gns and Ctrl group. The color key from blue to red indicates the relative gene expression level from low to high, respectively. $\mathbf{b}$ Volcano plot showing DEGs. The $x$-axis shows the foldchange in gene expression, and the $y$-axis shows significant statistical differences. Red, up-regulated genes; green, down-regulated genes; blue, genes with no significant difference in expression

that were down-regulated in the Gn-stimulated group are shown in Fig. 3a. Amongthem, only five pathways (cell cycle, oocyte meiosis, steroid hormone biosynthesis, P-mediated oocyte maturation, and ovarian steroidogenesis) were significantly enriched (adjusted $P<0.05$ ). The names of the DEGs in each pathway are annotated in Table 1. On the other hand, among the up-regulated genes in the Gn-stimulated group, 38 pathways were enriched (adjusted $P<0.05$ ). The top 10 terms are shown in Fig. 3b, including three representative terms (cytokine-cytokine receptor interaction, chemokine signaling pathway, and NF-kappa B signaling pathway), with the correlated input genes annotating in Table 1.

To further investigate whether oocyte meiosis was affected by $\mathrm{Gn}$ stimulation, we clustered some mitosis-associated genes and examined their expression among the seven samples (Fig. 4). This analysis revealed that some cell cycle- or mitosis-associated genes were expressed at low levels in the $\mathrm{Gn}$-stimulated group, including $C C N D 2$, $C C N B 1, C D C 23, C D K 1$, and SMAD3. Moreover, BUB3 (a mitotic checkpoint gene) was also expressed at low levels in the Gn-stimulated group. Intriguingly, HDAC2 (a member of the histone deacetylase family) was also down-regulated in the Gn-stimulated group, which forms transcriptional repressor complexes by associating with many different proteins, and thus plays an important role in transcriptional regulation, cell cycle progression, and developmental events.

\section{Hormonal concentrations in follicular fluid}

Next, we verified different hormone concentrations in the FF samples of every single pre-ovulatory follicle used for the transcriptome analysis. The sizes of the pre-ovulatory follicles were similar between the two groups. Table 2 shows the different follicular hormonal concentrations. The LH and E2 concentrations in FF were lower in the Gn-stimulated group than in the control group $(P=0.006$ and $P=0.002$, respectively). However, the P4 concentration was higher in the Gn-stimulated group $(P=0.003)$. The levels of the other four hormones tested were not significantly different between the two groups.

\section{Discussion}

In this paper, we compared the transcriptomes of granulosa cells from one leading pre-ovulatory follicle obtained from the control and Gn-stimulated groups. The results showed that DEGs down-regulated in the Gnstimulated group (COS-stimulated) were enriched in functions related to the cell cycle or mitosis, whereas up-regulated genes were mainly involved in immune functions and cytokine-cytokine receptor interactions.

Using a microarray technique, a previous study analyzed the transcriptomes of the cumulus cells obtained from human pre-ovulatory follicles in stimulated and unstimulated cycles and found that only 18 genes were significantly differentially expressed [13]. In that study, 


\section{A}

B

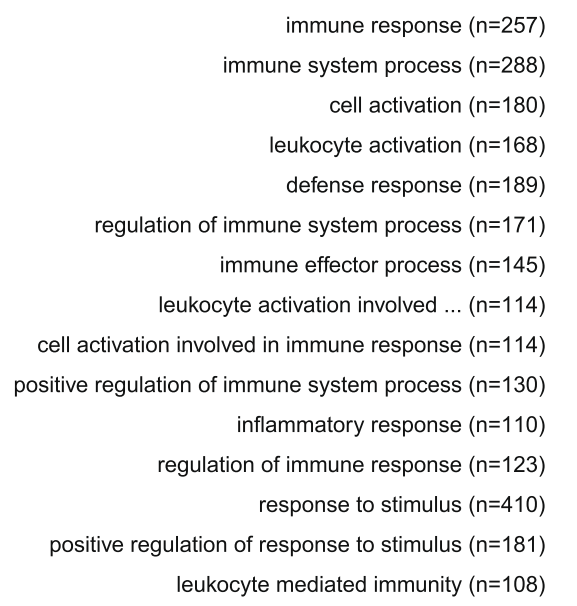

mitotic cell cycle process $(n=60)$

mitotic cell cycle $(n=63)$

cell cycle process $(n=69)$

chromosome segregation $(n=38)$

cell cycle $(n=77)$

cell division $(n=49)$

mitotic nuclear division $(n=33)$

nuclear division $(n=37)$

organelle fission $(n=37)$

nuclear chromosome segregation $(n=27)$

sister chromatid segregation $(n=23)$

mitotic sister chromatid segregation $(n=22)$

spindle $(n=30)$

chromosome, centromeric region $(n=24)$

cell cycle phase transition $(n=36)$

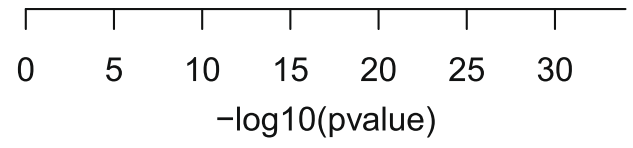

\section{GnsvsCtrl_up(GO)}

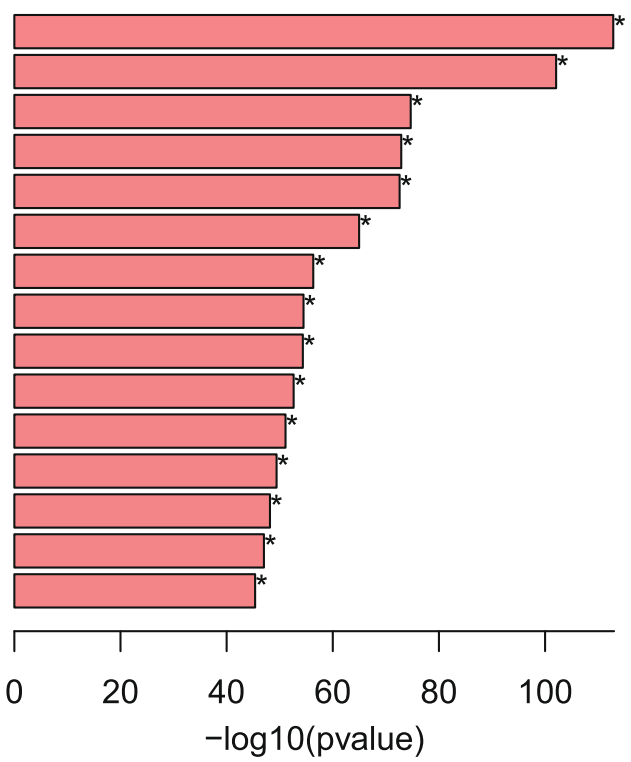

Fig. 2 GO enrichment in biological process analysis of DEGs. The x-axis shows - $\log _{10}(P$-value), and the $y$-axis shows five representative GO biological process terms and the number of genes representing each category ( $\mathrm{n})$. $\mathbf{a}$ Genes down-regulated in Gns compared with the Control; $\mathbf{b}$ Genes up-regulated in Gns compared with the Control

recombinant chorionic Gn (rCG) was administered to trigger ovulation during an unstimulated cycle. However, the control group assigned in our study did not apply any stimulation intervention, which represents a completely natural status. Moreover, we detected far more DEGs (715 up-regulated and 287 down-regulated genes) in the luteinized granulosa cells.
The expression levels of FSHR (FSH receptor), LHCGR (LH receptor), and INHBA (inhibin A) were decreased, which reflected a unique feature of the granulosa cells before and after ovulation triggered in the COS cycle [14]. The decreases in the expression levels of HSD11B2 (hydroxysteroid $11 \mathrm{~b}$ dehydrogenase 2 , which promotes cortisone production from cortisol), CYP171(which 


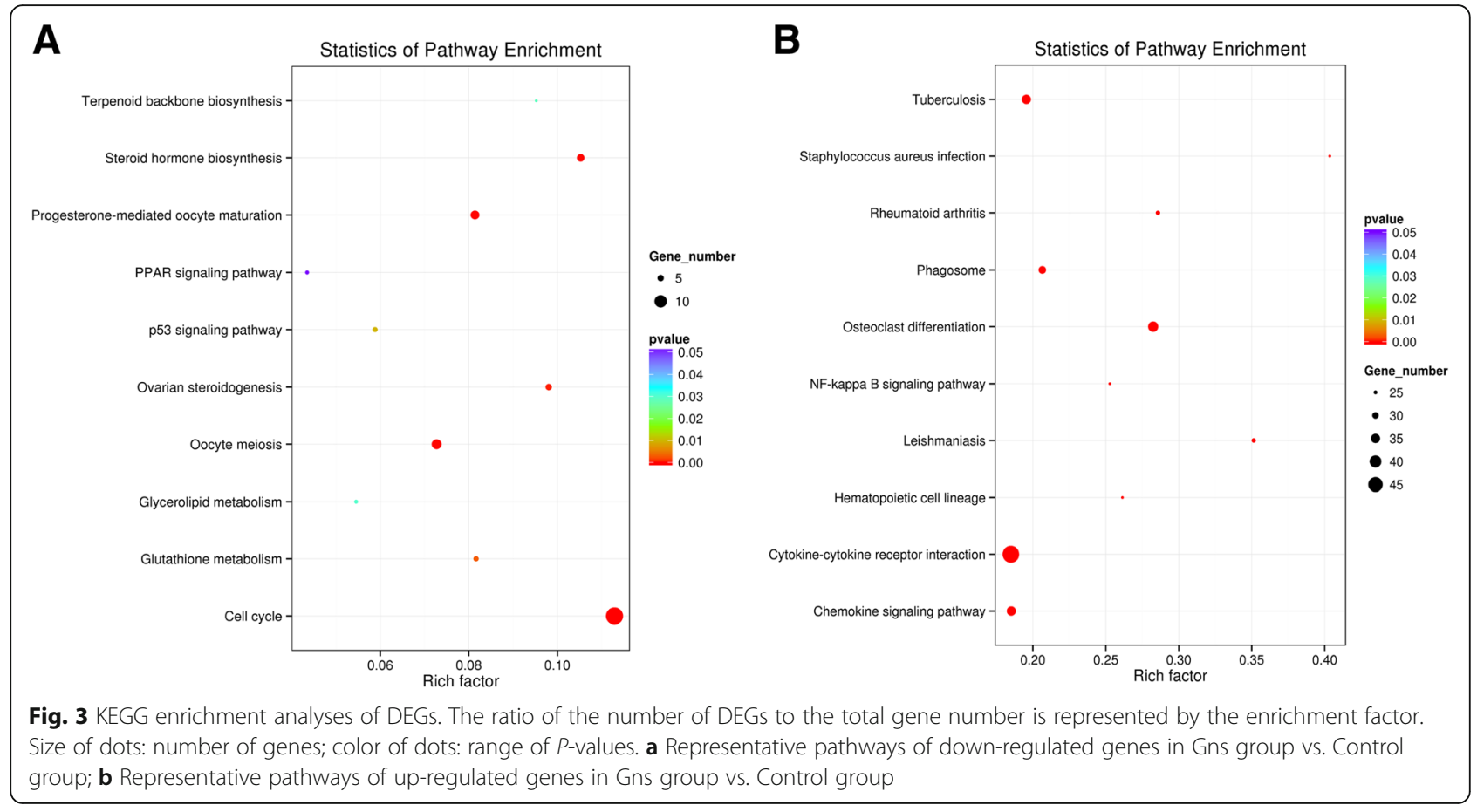

catalyzes androgen production), and CYP19A(which converts AND to estrogen) were accompanied by the changes in hormonal levels in FF, indicating a condition shifting from the estrogenic follicles to progesterogenic follicles under Gns stimulation.
The down-regulated genes were enriched in functions related to cell cycle andmiosis or meiosis, both of which are involved in the maturation processes of follicles and oocytes. This finding suggests that Gns can stimulate the maturation of granulosa cells by affecting the cell cycle

Table 1 Canonical pathways enriched $(P<0.001)$ among genes down-regulated or up-regulated in the Gns group versus the Control group

\begin{tabular}{|c|c|c|c|c|}
\hline Pathway & $\begin{array}{l}\text { Input } \\
\text { number }\end{array}$ & $\begin{array}{l}\text { Background } \\
\text { number }\end{array}$ & $\begin{array}{l}\text { Corrected } \\
P \text {-value }\end{array}$ & Genes \\
\hline \multicolumn{5}{|c|}{ Pathways enriched among down-regulated genes } \\
\hline Cell cycle & 14 & 124 & 1.10E-07 & PKMYT1, PLK1, CDC25A, TTK, CDC20, CDC25C, CCNA2, CCNB2, \\
\hline Oocyte meiosis & 8 & 110 & 0.003 & $\begin{array}{l}\text { BUB1B, CDC45, CCNB1, CDC6, ORC6, ESPL1 PKMYT1, PLK1, CDC20, } \\
\text { CDC25C, CCNB2, AURKA, CCNB1, ESPL1 }\end{array}$ \\
\hline $\begin{array}{l}\text { Steroid hormone } \\
\text { biosynthesis }\end{array}$ & 6 & 57 & 0.003 & CYP19A1, HSD11B2, CYP17A1, CYP21A2 \\
\hline $\begin{array}{l}\text { Progesterone-mediated } \\
\text { oocyte maturation }\end{array}$ & 7 & 86 & 0.003 & PKMYT1, PLK1, CDC25A, CCNB2, CDC25C, CCNA2, CCNB1 \\
\hline $\begin{array}{l}\text { Ovarian } \\
\text { steroidogenesis }\end{array}$ & 5 & 51 & 0.0125 & FSHR, CYP19A1, CYP17A1, LHCGR \\
\hline \multicolumn{5}{|c|}{ Pathways enriched among up-regulated genes } \\
\hline $\begin{array}{l}\text { Cytokine-cytokine } \\
\text { receptor interaction }\end{array}$ & 49 & 265 & $2.68 \mathrm{E}-10$ & $\begin{array}{l}\text { TNFSF11, CCL28, IL1B, CCR2, CXCL3, OSM, PPBP, TNF, IL10, TNFSF8, CCL4, CXCL8, } \\
\text { CXCR4, CD40, IL10RA, IL1R1, FLT3, CCR1, LTA, CCR5, TNFRSF11A, TGFBR2, CCL4L2, } \\
\text { TNFRSF18, CCL22, CXCL2, CCL8, TNFSF10, CCL2, CCL20, IL6R, CSF2RA, CCL3L1, } \\
\text { TNFSF14, TNFRSF8, TNFSF13B, IL1R2, IL6, CSF2RB, CCL3, CCR6, TNFRSF4, CRLF2, } \\
\text { CCL7, IL18R1, IL18RAP, TNFRSF1B, IL18, CSF3R }\end{array}$ \\
\hline $\begin{array}{l}\text { Chemokine signaling } \\
\text { pathway }\end{array}$ & 35 & 189 & $9.0 \mathrm{E}-08$ & $\begin{array}{l}\text { CCL28, WAS, CCR2, CXCL3, PRKCB, PPBP, CCL4, HCK, CXCL8, CXCR4, PIK3R5, } \\
\text { DOCK2, CCR1, CCR5, ARRB2, CCL4L2, CCL22, CXCL2, CCL8, CCL2, CCL20, } \\
\text { CCL3L1, ADCY7, CCR6, VAV1, ADCY4, FGR, CCL3, PIK3CG }\end{array}$ \\
\hline $\begin{array}{l}\text { NF-kappaB signaling } \\
\text { pathway }\end{array}$ & 23 & 91 & $1.4 \mathrm{E}-07$ & $\begin{array}{l}\text { CCL7, NCF1, PLCB2, GNGT2,TNFSF13B, PLAU, PRKCB, TNF, BLNK, BTK, CCL4, } \\
\text { CXCL8, CD40, LTA, BCL2A1, TNFRSF11A, CCL4L2, CXCL2, ICAM1, CD14, SYK, } \\
\text { TNFSF14, IL1B, IL1R1, TNFSF11, LY96, GADD45B }\end{array}$ \\
\hline
\end{tabular}




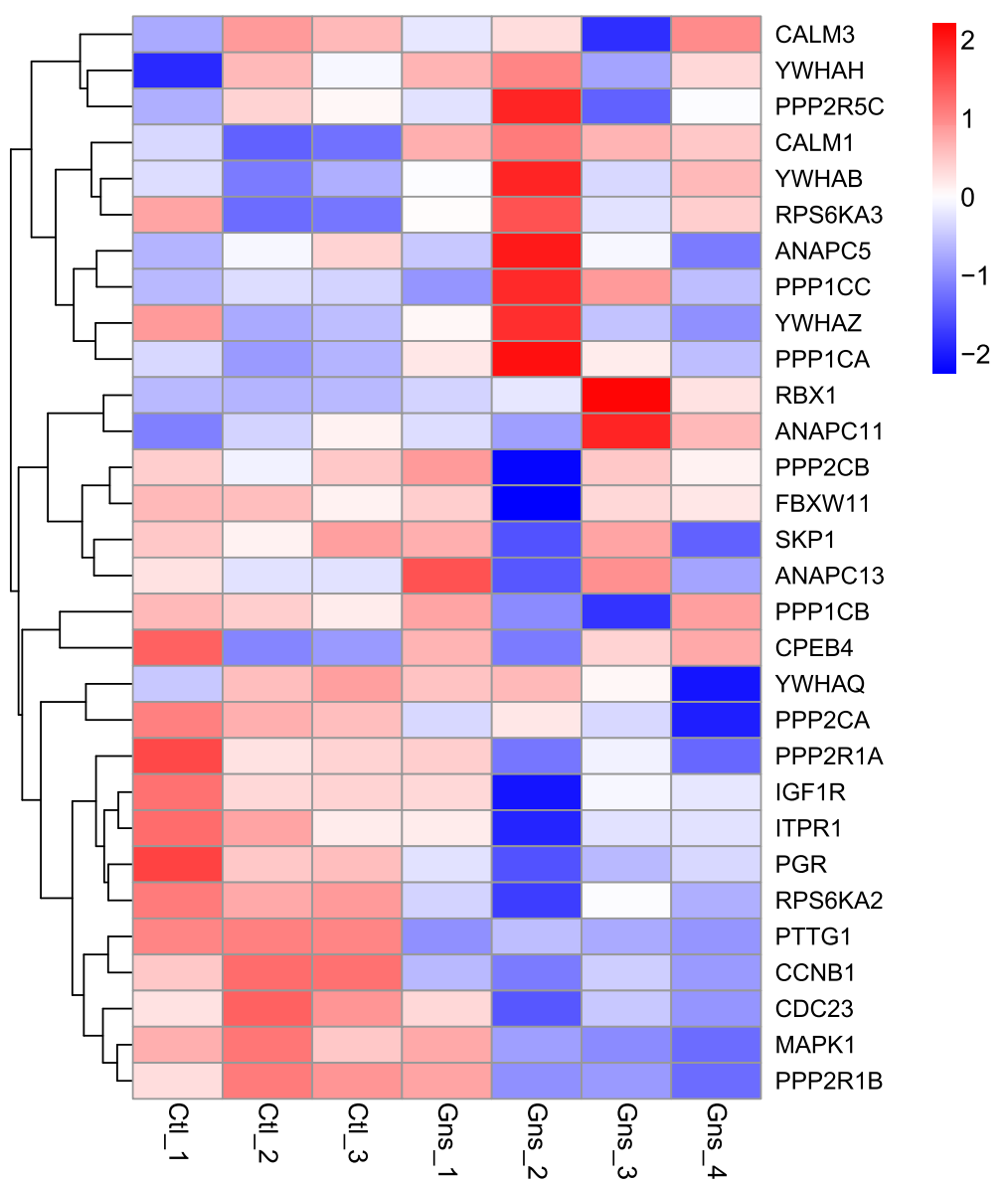

Fig. 4 Heatmap of selected genes involved in oocyte meiosis that were differentially expressed in the Control and Gns groups. Color from blue to red indicates the relative gene expression level from low to high

from the precocious maturation. These results implied adecrease in GC proliferation after Gns stimulation. The significant down-regulation of genes associated with chromosome segregation and the mitotic spindle is consistent with the concept that Gns increase aneuploidy in oocytes [15] and spindle abnormalities [13]. For example, PRC1 (a protein regulator of cytokinesis 1) was down-

Table 2 Comparison of hormonal concentration in follicular fluid aspirated respectively from the Gns and Control groups

\begin{tabular}{llll}
\hline Parameters & Control & Gns & P-value \\
\hline Follicular diameter $(\mathrm{cm})$ & $1.8 \pm 0.2$ & $2.0 \pm 0.1$ & $\mathrm{~ns}$ \\
FSH $(\mathrm{mlU} / \mathrm{ml})$ & $1.7 \pm 1.0$ & $7.4 \pm 7.3$ & $\mathrm{~ns}$ \\
$\mathrm{LH}(\mathrm{mlU} / \mathrm{ml})$ & $4.7 \pm 1.7$ & $1.0 \pm 0.3$ & $<0.01$ \\
E2 $(\mathrm{nmol} / \mathrm{l})$ & $5970.4 \pm 910.5$ & $1119.5 \pm 1088.4$ & $<0.01$ \\
P4 $(\mu \mathrm{mol} / \mathrm{l})$ & $7.3 \pm 6.5$ & $25.4 \pm 2.4$ & $<0.01$ \\
T $(\mathrm{nmol} / \mathrm{l})$ & $43.7 \pm 27.2$ & $12.3 \pm 5.3$ & $\mathrm{~ns}$ \\
AND $(\mathrm{nmol} / \mathrm{l})$ & $383.7 \pm 189.8$ & $163.4 \pm 120.8$ & $\mathrm{~ns}$ \\
\hline
\end{tabular}

$\mathrm{mIU} / \mathrm{ml}$, milli-international units per milliliter

All the values are means \pm SD (standard deviation). $P$-values were calculated using Student's t test. ns not significant regulated, which potentially impairs the completion of the first meiotic division [16]. Taken together, these observations confirmed the concept that granulosa cells grow more quickly when approaching ovulation but may have been insufficiently mature after Gns stimulation.

On the other hand, the up-regulation of immune and inflammation-associated genes in the Gn-stimulated group was consistent with the theory that ovulation is an acute inflammatory reaction occurs in the ovarian tissue [17]. Consistent with the results obtained from previous studies, several ovulation-associated factors such as PTGS1 (prostaglandin endoperoxide synthetase 1), RGS1, RGS16 (a regulator of G-protein signaling 1 and 16),PDE2A (3'5'-cyclic nucleotide phosphodiesterase 2A), and ADAMTS1 (a disintegrin-like and metalloprotease with thrombospondin type 1 motif, 1) were all upregulated in our study $[14,18]$.

Immune cell-derived cytokines and chemokines play important roles in regulating ovarian function [19]. Gns induce the local distribution of immune cells to release intrafollicular cytokines, which may then, in turn, affect the oocyte quality [20]. Levels of cytokines such as IL1 $\beta$, 
IL6, and TNF- $\alpha$ were elevated in FFfollowing controlled ovarian hyperstimulation $(\mathrm{COH})$ [21]. In the present study, we also observed significantly up-regulated expression levels of IL10, IL6, IL18, and TNF. Cytokinecytokine receptor interactions and the TNF signaling pathway were all functionally enriched among upregulated DEGs. Inconsistent with the results obtained from a previous study, Baskindet al. [22], reported that the majority of circulatory cytokines, such as IL8, were present at higher concentrations in the modified natural-cycle cohort than in the $\mathrm{COH}$ group. Kollmann et al. [20] also reported that FF contained a marginally lower concentration of IL8 under a stimulated cycle than a natural cycle. The reason for this discrepancy in these two studies may be that HCG was administered to their natural-cycle cohorts.

Two previous studies have compared the FF hormone profiles of the natural IVF cycle (with HCG administration) and the conventional stimulated IVF cycle and found that E2 and LH concentrations were significantly reduced in the $\mathrm{Gn}$-stimulated group, which is consistent with our results $[13,23]$. However, HCG was administered in the natural-cycle group as in both studies performed by de Los et al.and von Wolff $\mathrm{M}$ et al. Similar to the finding that there is a change before and after ovulation triggered using HCG, the concentrations of E2 and $\mathrm{P} 4$ were different between the two groups [14]. However, two other studies reported that E2 and P4 levels in FF did not differ significantly between stimulated and natural groups, in whichno HCG was administered to the natural-cycle group [21, 24]. In particular, we have to point out that we analyzed only the largest follicle of a population of multiple follicles, which was the clearest and free from contamination (especially blood cells). Therefore, we assure that the results of the hormonal measurement and granulosa cells transcriptome are sound.

There are some limitations throughout the current study. First, the transcriptome data were obtained from the mural granulosa cells obtained from FF, but not the cells that surround the oocyte, even though some DEGs were correlated with oocyte meiosis. Second, we aimed to observe the influence of Gns, however we were not able to exclude the effect of HCG administration, which was applied only in the Gn-stimulated group. On the other hand, data obtained from the natural cycle without hCG triggering may reflect a more physiological status and hence more meaningful. Third, we have to admit that the present sample size was limited and small, even though some of the results are conclusive.

\section{Conclusions}

In summary, using a global transcriptome sequencing technique, we compared a set of genes that were differentially regulated in granulosa cells of the maturation processes in natural and $\mathrm{Gn}$-stimulated cycles. The results obtained from the DEGs analyses suggested that the Gn stimulation may induce the decreased proliferation of granulosa cells, ovulation, and an increased differentiation process. Additionally, these changes were accompanied by the alteration of several hormonal levels in FF. These findings may improve our understanding of the maturation process of the oocyte. Most importantly, our findings indicate that the simulation with Gns may potentially induce meiotic errors of human oocytes.

\section{Additional files}

Additional file 1: Figure S1. Correlation and gene expression analysis. A, Pearson correlation between samples; B, Venn diagram showing overlaps between the two groups. Purple, genes expressed distinctly in the Gns group; yellow, genes expressed distinctly in the Control group; orange, genes expressed in both groups. (PDF $627 \mathrm{~kb}$ )

Additional file 2: Table S1. Summary of sequence assembly after Illumina sequencing. (DOCX $15 \mathrm{~kb}$ )

Additional file 3: Table S2. Details of genes down-regulated in the Gns group compared with the Control group. (XLSX $36 \mathrm{~kb}$ )

Additional file 4: Table S3. Details of genes up-regulated in the Gns group compared with the Control group. (XLSX 86 kb)

Additional file 5: Table S4. GO enrichment of genes down-regulated in the Gns group compared with the Control group. (XLSX $420 \mathrm{~kb}$ )

Additional file 6: Table S5. GO enrichment of genes up-regulated in the Gns group compared with the Control group. (XLSX 791 kb)

Additional file 7: Table S6. Pathway enrichment of genes downregulated in the Gns group compared with the Control group. (XLSX 26 kb)

Additional file 8: Table S7. Pathway enrichment of genes up-regulated in the Gns group compared with the Control group. (XLSX $45 \mathrm{~kb}$ )

\section{Acknowledgements}

We sincerely thank Dr. Hsun-Ming Chang, Department of Obstetrics and Gynaecology, BC Children's Hospital Research Institute, University of British Columbia, Vancouver, British Columbia, Canada, for his insightful comments and language guidance. We sincerely thank the women who participated in the study. We also thank the medical and nursing staff of the Reproductive Medical Center of Peking University Third Hospital for their assistance in patient recruitment and management.

\section{Authors' contributions}

$\mathrm{RL}, \mathrm{PL}, \mathrm{HLF}$, and JQ designed the study, directed the study, and revised the manuscript. CLL processed the samples, contributed to sequencing, and wrote the paper. ZQY conducted the bioinformatics analysis. XLS collected the granulosa cells. YYX performed the hormone measurements. XYZ involved in the paper writing. All authors read and approved the final manuscript.

\section{Funding}

The study was supported by the Joint Research Fund for Overseas Natural Science of China (grant no. 31429004), Special fund for Reproductive Medical Research of Chinese Medical Association (16020400656), and the National Natural Science Foundation of China (grant no. 81521002 and 81730038).

\section{Availability of data and materials}

All data generated or analysed during this study are included in this published article [and its supplementary information files]. 


\section{Ethics approval and consent to participate}

The study was approved by the institutional review board of Peking University Third Hospital. All participants signed an informed consent form to participate in the study.

\section{Consent for publication}

Not applicable.

\section{Competing interests}

The authors declare that they have no competing interests.

\section{Author details}

${ }^{1}$ Center for Reproductive Medicine, Department of Obstetrics and Gynecology, Peking University Third Hospital, Beijing 100191, China. ${ }^{2}$ National Clinical Research Center for Obstetrics and Gynecology, Beijing 100191, China. ${ }^{3}$ Key Laboratory of Assisted Reproduction, Ministry of Education, Peking University, Beijing 100191, China. ${ }^{4}$ Beijing Key Laboratory of Reproductive Endocrinology and Assisted Reproductive Technology, Beijing 100191, China. ${ }^{5}$ Peking-Tsinghua Center of Life Sciences, Peking University, Beijing 100871, China. 'Beijing Advanced Innovation Center for Genomics, Beijing 100871, China. ${ }^{7}$ Department of Obstetrics and Gynecology, New York Hospital Queens-affiliated Weill Medical College, Cornell University, New York 10041NY212, USA.

Received: 18 January 2019 Accepted: 24 May 2019

Published online: 24 June 2019

\section{References}

1. Lindenberg S, Almind GJ, Lindenberg FB. Is gonadotropin stimulation bad for oocytes? Curr Opin Obstet Gynecol. 2018;30(3):151-4.

2. Macklon NS, Stouffer RL, Giudice LC, Fauser BC. The science behind 25 years of ovarian stimulation for in vitro fertilization. Endocr Rev. 2006; 27(2):170-207.

3. Baart EB, Martini E, Eijkemans MJ, Van Opstal D, Beckers NG, Verhoeff A, Macklon NS, Fauser BC. Milder ovarian stimulation for in-vitro fertilization reduces aneuploidy in the human preimplantation embryo: a randomized controlled trial. Hum Reprod. 2007:22(4):980-8.

4. Munne S, Magli C, Adler A, Wright G, de Boer K, Mortimer D, Tucker M, Cohen J, Gianaroli L. Treatment-related chromosome abnormalities in human embryos. Hum Reprod. 1997;12(4):780-4.

5. Sutton ML, Gilchrist RB, Thompson JG. Effects of in-vivo and in-vitro environments on the metabolism of the cumulus-oocyte complex and its influence on oocyte developmental capacity. Hum Reprod Update. 2003; 9(1):35-48.

6. Van der Auwera I, D'Hooghe T. Superovulation of female mice delays embryonic and fetal development. Hum Reprod. 2001;16(6):1237-43.

7. Lee M, Ahn Jl, Lee AR, Ko DW, Yang WS, Lee G, Ahn JY, Lim JM. Adverse effect of superovulation treatment on maturation, function and ultrastructural integrity of murine oocytes. Mol Cells. 2017;40(8):558-66.

8. Xu GF, Zhang JY, Pan HT, Tian S, Liu ME, Yu TT, Li JY, Ying WW, Yao WM, $\mathrm{Lin} X \mathrm{XH}$, et al. Cardiovascular dysfunction in offspring of ovarianhyperstimulated women and effects of estradiol and progesterone: a retrospective cohort study and proteomics analysis. J Clin Endocrinol Metab. 2014;99(12):E2494-503.

9. Xu GF, Zhou CL, Xiong YM, Li JY, Yu TT, Tian S, Lin XH, Liao Y, Lv Y, Zhang $\mathrm{FH}$, et al. Reduced intellectual ability in offspring of ovarian Hyperstimulation syndrome: a cohort study. EBIOMEDICINE. 2017;20:263-7.

10. Picelli S, Bjorklund AK, Faridani OR, Sagasser S, Winberg G, Sandberg R. Smart-seq2 for sensitive full-length transcriptome profiling in single cells. Nat Methods. 2013;10(11):1096-8.

11. Trapnell C, Williams BA, Pertea G, Mortazavi A, Kwan G, van Baren MJ, Salzberg SL, Wold BJ, Pachter L. Transcript assembly and quantification by RNA-Seq reveals unannotated transcripts and isoform switching during cell differentiation. Nat Biotechnol. 2010;28(5):511-5.

12. Subramanian A, Tamayo P, Mootha VK, Mukherjee S, Ebert BL, Gillette MA, Paulovich A, Pomeroy SL, Golub TR, Lander ES, et al. Gene set enrichment analysis: a knowledge-based approach for interpreting genome-wide expression profiles. Proc Natl Acad Sci U S A. 2005:102(43):15545-50.

13. de Los SM, Garcia-Laez V, Beltran-Torregrosa D, Horcajadas JA, MartinezConejero JA, Esteban FJ, Pellicer A, Labarta E. Hormonal and molecular characterization of follicular fluid, cumulus cells and oocytes from pre- ovulatory follicles in stimulated and unstimulated cycles. Hum Reprod. 2012; 27(6):1596-605.

14. Wissing ML, Kristensen SG, Andersen CY, Mikkelsen AL, Host T, Borup R, Grondahl ML. Identification of new ovulation-related genes in humans by comparing the transcriptome of granulosa cells before and after ovulation triggering in the same controlled ovarian stimulation cycle. Hum Reprod. 2014;29(5):997-1010.

15. Xu YW, Peng YT, Wang B, Zeng YH, Zhuang GL, Zhou CQ. High folliclestimulating hormone increases aneuploidy in human oocytes matured in vitro. Fertil Steril. 2011;95(1):99-104.

16. Guo J, Zhang T, Guo Y, Sun T, Li H, Zhang X, Yin H, Cao G, Yin Y, Wang H, et al. Oocyte stage-specific effects of MTOR determine granulosa cell fate and oocyte quality in mice. Proc Natl Acad Sci U S A. 2018

17. Espey LL. Comprehensive analysis of ovarian gene expression during ovulation using differential display. Methods Mol Biol. 2006;317:219-41.

18. Hennebold JD. Characterization of the ovarian transcriptome through the use of differential analysis of gene expression methodologies. Hum Reprod Update. 2004;10(3):227-39.

19. Qiao J, Feng HL. Extra- and intra-ovarian factors in polycystic ovary syndrome: impact on oocyte maturation and embryo developmental competence. Hum Reprod Update. 2011;17(1):17-33.

20. Kollmann Z, Schneider S, Fux M, Bersinger NA, von Wolff M. Gonadotrophin stimulation in IVF alters the immune cell profile in follicular fluid and the cytokine concentrations in follicular fluid and serum. Hum Reprod. 2017; 32(4):820-31.

21. Loret DMJ, Goldfarb JM, Hecht BR, Baumgardner GP, Babbo CJ, Friedlander MA. Gonadotropins induce the release of interleukin-1 beta, interleukin-6 and tumor necrosis factor-alpha from the human preovulatory follicle. Am J Reprod Immunol. 1998;39(6):387-90.

22. Baskind NE, Orsi NM, Sharma V. Impact of exogenous gonadotropin stimulation on circulatory and follicular fluid cytokine profiles. Int J Reprod Med. 2014;2014:218769.

23. von Wolff M, Kollmann Z, Fluck CE, Stute P, Marti U, Weiss B, Bersinger NA. Gonadotrophin stimulation for in vitro fertilization significantly alters the hormone milieu in follicular fluid: a comparative study between natural cycle IVF and conventional IVF. Hum Reprod. 2014;29(5):1049-57.

24. Loret DMJ, Goldfarb JM, Hecht BR, Babbo CJ, Friedlander MA. Gonadotropins induce higher active renin levels in the follicular fluid of normal and hyperstimulated cycles. Gynecol Endocrinol. 1999;13(3):155-60.

\section{Publisher's Note}

Springer Nature remains neutral with regard to jurisdictional claims in published maps and institutional affiliations.

\section{Ready to submit your research? Choose BMC and benefit from:}

- fast, convenient online submission

- thorough peer review by experienced researchers in your field

- rapid publication on acceptance

- support for research data, including large and complex data types

- gold Open Access which fosters wider collaboration and increased citations

- maximum visibility for your research: over $100 \mathrm{M}$ website views per year

At $\mathrm{BMC}$, research is always in progress.

Learn more biomedcentral.com/submissions 